\title{
Downregulation of microRNA-451 improves cell migration, invasion and tube formation in hypoxia-treated HUVECs by targeting MIF
}

\author{
QIAN LI, YONGQIU LI, DONGSEN ZHANG, HAIFENG GAO and XUAN GAO
}

Department of Neurology, The Workers' Hospital of Tangshan City, Tangshan, Hebei 063000, P.R. China

Received May 22, 2018; Accepted January 4, 2019

DOI: $10.3892 / \mathrm{mmr} .2019 .10357$

\begin{abstract}
Angiogenesis is a critical process of recovery from cerebrovascular disease. A growing body of evidence has confirmed that microRNAs (miRNAs/miRs) have an important role in the modulation of angiogenesis under physiological and pathological conditions including cerebral ischemia injury (CII). Therefore, the aim of the present study was to explore the function and mechanism of microRNAs in regulating angiogenesis using a cell model of CII. Firstly, a miRNA microarray was performed to analyze miRNA expression in serum samples from patients with cerebral ischemia and the results revealed that miR-451 was one of the miRNAs that was the most significantly downregulated. Subsequently, human umbilical vein endothelial cells (HUVECs) were used as an in vitro model to further explore the mechanisms governing angiogenesis during hypoxia. The results demonstrated that overexpression of miR-451 had a significantly anti-angiogenic effect by suppressing tube formation, migration and wound healing in vitro. By contrast, reducing the expression of miR-451 promoted HUVEC migration and tubulogenesis under normoxic conditions. The present study further identified that macrophage migration inhibitory factor (MIF), an important angiogenic regulator, was a novel target of miR-451 that could reverse the effects of miR-451 on the regulation of angiogenesis in HUVECs under hypoxic or normoxic conditions. These results revealed that downregulation of miR-451 promotes angiogenesis by targeting MIF in hypoxic HUVECs and indicated that miR-451 is a potential candidate for CII therapeutics.
\end{abstract}

\section{Introduction}

Ischemic stroke is a common clinical disorder that affects the global population with high incidence, mortality and disability $(1,2)$. Cerebral ischemic injury (CII) caused by

Correspondence to: Dr Qian Li, Department of Neurology, The Workers' Hospital of Tangshan City, 27 Wenhua Road, Lubei, Tangshan, Hebei 063000, P.R. China

E-mail: qianliq122@163.com

Key words: angiogenesis, cerebral ischemia injury, microRNA-451, macrophage migration inhibitory factor cerebral ischemia is the main pathological and physiological basis of ischemic stroke (3). Angiogenesis is an important compensatory mechanism following ischemic stroke, which has been implicated in animal models and human patients (4). However, this mechanism is not enough to attenuate CII. Thus, understanding the mechanism controlling angiogenesis is of significance for the development of effective therapeutic strategies for ischemic stroke.

Macrophage migration inhibitory factor (MIF), one of the first functional cytokines identified, is an important angiogenic regulator $(5,6)$. Rassaf et al (7) demonstrated that MIF upregulation improved angiogenesis in myocardial ischemia/reperfusion injury. Liao et al (8) reported that MIF contributed to angiogenesis by upregulating interleukin (IL)- 8 in primary nasopharyngeal carcinoma. Girard et al (9) demonstrated that overexpression of MIF is involved in angiogenesis in the B16-F10 melanoma model, and the absence of MIF resulted in slower tumor growth, which was associated with reduced vascularity. An accumulating body of evidence has indicated that MIF is overexpressed during ischemic stroke in patients and a rat stroke model, and was associated with the severity of the pathology $(10,11)$. However, how MIF works in CII remains unknown.

MicroRNAs (miRNAs/miRs) are small endogenous non-coding RNAs that negatively regulate gene expression by binding to the 3'-untranslated region (UTR) of target mRNAs $(12,13)$. Several miRNAs have been identified to be involved in the regulation of angiogenesis. For example, Liu et al (14) revealed that miR-106b and miR-15b modulate angiogenesis in myocardial infarction. Downregulation of miR-195 promoted angiogenesis induced by cerebral infarction by targeting vascular endothelial growth factor A (VEGFA) (15). In addition, Li et al (16) revealed that miR-493 inhibited tube formation and the migration of rat brain microvascular endothelial cells by suppressing MIF. However, limited studies have focused on the functions of miRNAs in the regulation of angiogenesis following cerebral ischemia.

The present study performed a miRNA microarray to investigate miRNA expression in the serum samples of cerebral ischemic patients. Then, the roles and underlying mechanisms of the candidate miRNA, miR-451, in the regulation of angiogenesis were investigated using a cell model of CII. The present results indicate that miR-451 may be a potential therapeutic option for CII. 


\section{Materials and methods}

Serum samples. Serum samples were obtained from 15 patients with cerebral ischemia who were also diagnosed with ischemic stroke by MRI, as well as 15 healthy participants at the Workers' Hospital of Tangshan City (Hebei, China). All experimental protocols were approved by the Ethics Committee of the Workers' Hospital of Tangshan City. Written informed consent was obtained from all patients. All samples were flash-frozen in liquid nitrogen, and stored at $-80^{\circ} \mathrm{C}$ until further molecular analysis. The demographics and clinical characteristics of the 15 cerebral ischemic patients and 15 healthy controls are provided in Table I.

miRNA microarray. Total RNA was isolated from the sera of patients with cerebral ischemia by a miRNAeasy mini kit (Qiagen, Inc., Valencia, CA, USA). The purity and quantity of total RNA were evaluated by NanoDrop ND-1000 Spectrophotometry (Thermo Fisher Scientific, Inc., Waltham, MA, USA) and Agilent's 2100 Bioanalyzer (Agilent Technologies, Inc., Santa Clara, CA, USA). Total RNA $(200 \mathrm{ng})$ was labeled and hybridized with the miRCURY ${ }^{\mathrm{TM}}$ LNA Array (version 16.0; Exiqon; Qiagen, Inc.). Following washing, Axon GenePix 4000B microarray scanner (Axon Instruments; Molecular Devices, LLC, Sunnyvale, CA, USA) was used to scan the fluorescence intensity of the microarray. Scanned images were then imported into the GenePix Pro6.0 program (Axon Instruments; Molecular Devices, LLC) for grid alignment and data extraction. Finally, the heat map of the 57 miRNAs with the most evident differences was created using a method of hierarchical clustering with GeneSpring GX, version 7.3 (Agilent Technologies, Inc.).

Cell culture and hypoxia. HUVECs were obtained from the cell bank of the Chinese Academy of Sciences (Shanghai, China), and maintained in M199 medium supplemented with $20 \mathrm{mg} / \mathrm{ml}$ endothelial cell growth supplement (Upstate Biotechnology, Inc., Lake Placid, NY, USA) and 10\% fetal bovine serum (FBS; HyClone; GE Healthcare Life Sciences, Logan, UT, USA) at $37^{\circ} \mathrm{C}$ in a humidified incubator with an atmosphere of $95 \%$ air and $5 \% \mathrm{CO}_{2}$ (normoxic conditions). For hypoxia, HUVECs were cultured in a hypoxia incubator (Sanyo Electric Co., Ltd., Osaka, Japan) under hypoxic conditions $\left(5 \% \mathrm{CO}_{2}, 94 \% \mathrm{~N}_{2}\right.$ and $1 \% \mathrm{O}_{2}$,) for $6 \mathrm{~h}$ at $37^{\circ} \mathrm{C}$. Cells cultured under normoxic conditions were used as controls.

Reverse transcription-quantitative polymerase chain reaction (RT-qPCR). Total RNA was isolated from serum samples and HUVECs cells using TRIzol reagents (Invitrogen; Thermo Fisher Scientific, Inc.) following the manufacturer's instructions and reverse transcription was performed using PrimeScript ${ }^{\mathrm{TM}}$ RT reagent kit (Takara Biotechnology Co., Ltd., Dalian, China) at $42^{\circ} \mathrm{C}$ for $30 \mathrm{~min}$ and $85^{\circ} \mathrm{C}$ for $5 \mathrm{sec}$. For the detection of miRNA, RT-qPCR assays were performed using the TaqMan miRNA Assay (Thermo Fisher Scientific, Inc.) following the manufacturer's instructions. For detection of the mRNA levels, qPCR was performed on an ABI PRISM 7300 sequence detection system in an SYBR Green I Real-Time PCR kit (Applied Biosystems; Thermo Fisher Scientific, Inc.).
The RT-qPCR reaction system (30 $\mu \mathrm{l})$ contained $5 \mu \mathrm{l}$ cDNA, $15 \mu 12 \mathrm{X}$ qPCR mix, $1 \mu 1$ upstream primer, $1 \mu \mathrm{l}$ downstream primer and $8 \mu \mathrm{l}$ double distilled $\mathrm{H}_{2} \mathrm{O}$. The PCR protocol was: $95^{\circ} \mathrm{C}$ for $15 \mathrm{~min}$, followed by 40 cycles of $94^{\circ} \mathrm{C}$ for $15 \mathrm{sec}$, $55^{\circ} \mathrm{C}$ for $30 \mathrm{sec}$ and $70^{\circ} \mathrm{C}$ for $30 \mathrm{sec}$ and a final extension step at $72^{\circ} \mathrm{C}$ for $5 \mathrm{~min}$. U6 and GAPDH functioned as the normalization controls in the expression analysis of miR-451 and MIF, respectively. The relative expression of RNAs was calculated using the $2^{-\triangle \Delta C a}$ method (17). Each reaction was conducted in triplicate. The primers utilized for RT-qPCR analysis were as follows: miR-451 forward, 5'-AAAGTCGACAAGCTCTCT GCTCAGCCTGTC-3' and reverse, 5'-AAAATATCTCGA GCCCCCACCCCTGCCTTGT-3'; U6 forward, 5'-TGCGGG TGCTCGCTTCGCAGC-3' and reverse, 5'-CCAGTGCAG GGTCCGAGGT-3'; MIF forward, 5'-GGCCTCACTTACCTG CACC-3' and reverse, 5'-AACCATTTATTTCTCCCGACC-3'; GAPDH forward, 5'-GCAACTCCCACTCTTCCACC-3' and reverse, 5'-GTCATACCAGGAAATGAGCTTGACA-3'. The RT-qPCR assays were performed in triplicate and the change in expression level was calculated using the $2^{-\Delta \Delta C q}$ method.

Cell transfection. HUVECs were seeded at a density of $6 \times 10^{4} / \mathrm{cm}^{2}$ in 6-well plates and allowed to settle for $24 \mathrm{~h}$ to ensure that $40-50 \%$ confluence was achieved prior to transfection. miR-451 mimic (5'-AAACCGUUACCAUUACUG AGUU-3') and its negative control (NC; 5'-UCGCUUGGU GCAGGUCGGGAA-3'), miR-451 inhibitor (5'-AACUCA GUAAUGGUAACGGUUU- $3^{\prime}$ ) and negative control (NC; 5'-CAGUACUUUUGUGUAGUACAA-3') were synthesized by Shanghai GenePharma Co., Ltd. (Shanghai, China). The MIF small interfering RNA (siRNA, (5'-ACACCAACGUGC CCCGCGCdTdT-3') and NC siRNA (5'-GCGCGGGGC ACGUUGGUGUdTdT-3') were purchased from Santa Cruz Biotechnology, Inc. (Dallas, CA, USA). In addition, to enhance the expression of MIF, the coding domain sequences of MIF mRNA were amplified by PCR, and inserted into pcDNA 3.0 vector (Invitrogen; Thermo Fisher Scientific, Inc.), and named pcDNA-MIF. Cells were cultured to $80 \%$ confluence, followed by transfection with miR-451 mimics $(50 \mathrm{nM})$, control mimics, miR-451 inhibitor $(50 \mathrm{nM})$ or control inhibitor using Lipofectamine $2000^{\circledR}$ (Invitrogen; Thermo Fisher Scientific, Inc.) according to the manufacturer's instructions. MIF expressing vector $(2 \mu \mathrm{g})$ was transfected into HUVECs with Lipofectamine ${ }^{\circledR} 2000$ following manufacturer's protocol. After $24 \mathrm{~h}$, the transfected cells were subjected to hypoxic conditions for $6 \mathrm{~h}$. The transfection efficiency was determined by RT-qPCR.

Tube formation. Confluent HUVEC monolayers were $0.25 \%$ trypsinized and plated onto 24 -well plates that were coated with Matrigel (Becton, Dickinson and Company, Franklin, Lakes, NJ, USA) and incubated in M199 medium for $24 \mathrm{~h}$ at $37^{\circ} \mathrm{C}$. Then, the transfected cells were subjected to hypoxic conditions for $6 \mathrm{~h}$ as aforementioned and tube-like structures were observed under a routine light microscope and images captured from five randomly selected microscopic fields. Tube formations were calculated by counting the number of branches, which was conducted using ImageJ software (Version 1.46, National Institutes of Health, Bethesda, MD, USA). 
Transwell invasion assay. Invasion activities of HUVECs were analyzed using Boyden chambers with $8-\mathrm{mm}$ pore membranes coated with Matrigel (Becton, Dickinson and Company) following manufacturer's protocol. Briefly, following $24 \mathrm{~h}$ transfection, HUVECs $\left(1 \times 10^{4}\right.$ per well) were seeded into the upper chambers in $200 \mu \mathrm{l}$ serum-free M199 medium, and the lower chambers were filled with $500 \mu \mathrm{l}$ of M199 medium containing 10\% FBS. Following exposure to hypoxic or normoxic conditions for $6 \mathrm{~h}$, cells on the surface of the filter were fixed with $4 \%$ formaldehyde, stained with $0.5 \%$ crystal violet at $37^{\circ} \mathrm{C}$ for $30 \mathrm{~min}$, and counted under a routine light microscope (Phenix Optical Instrument Group Company, Jiangxi, China). Cells were counted in five randomly chosen microscopic fields.

Wound healing assay. HUVECs $\left(1 \times 10^{6}\right.$ per well) were plated onto 6-well plates and incubated in M199 for $24 \mathrm{~h}$ at $37^{\circ} \mathrm{C}$. Following $24 \mathrm{~h}$ transfection, the cells were scrapped with a $10 \mu l$ pipette tip, fresh serum-free medium was added and then cells were exposed to hypoxic or normoxic conditions for $6 \mathrm{~h}$. Initial images were acquired as a reference and, following $6 \mathrm{~h}$ secondary images were taken corresponding to the photographed region capture initially. Wound healing was evaluated by measuring the distance of the wounded region with an absence of cells using ImageJ software (Version 1.46, National Institutes of Health, Bethesda, MD, USA).

Bioinformatics. TargetScan (version 7.0; www.targetscan. org/) and PicTar (version 2006; https://pictar.mdc-berlin.de) target gene prediction software were used to select MIF as a target gene of miR-451.

Luciferase reporter assay. The 3'-untranslated region (UTR) of MIF and the mutated sequence were inserted into the pGL3 control vector (Promega Corporation, Madison, WI, USA) to construct the wild-type (wt) MIF-3'-UTR vector and mutant MIF-3'-UTR vector, respectively. For the luciferase reporter assay, HUVECs were transfected with the corresponding vectors using Lipofectamine $2000{ }^{\circledR}$ (Invitrogen; Thermo Fisher Scientific, Inc.); a 48 h post-transfection, the dual-luciferase reporter assay system (Promega Corporation) was used to measure luciferase activity. To correct for differences in transfection and harvesting efficiencies, Renilla luciferase activity was used to normalize the firefly luciferase activity. All experiments were performed in triplicate.

Western blot analysis. Total protein was extracted from HUVECs cells using radioimmunoprecipitation lysis buffer (Beyotime Institute of Biotechnology, Shanghai, China). Concentrations of total cellular protein were determined using a BCA assay kit (Pierce; Thermo Fisher Scientific, Inc.). Total protein samples $(40 \mu \mathrm{g})$ were analyzed by $8 \%$ SDS-PAGE and transferred to polyvinylidene difluoride membranes (GE Healthcare, Chicago, IL, USA) by electroblotting. Membranes were blocked with $5 \%$ nonfat milk at room temperature for $1 \mathrm{~h}$, followed by incubation overnight at $4^{\circ} \mathrm{C}$ with primary antibodies. Primary antibodies against MIF (cat. no. sc-130329; Santa Cruz Biotechnology, Inc., Danvers, MA, USA; 1:1,000 dilution), phospho (p)-VEGF Receptor 2 (cat. no. 2478; VEGFR2; Tyr1175; Cell Signaling Technology, Inc.; 1:1,000
Table I. Demographic and clinical characteristics in patients with cerebral ischemia and normal controls.

\begin{tabular}{lcc}
\hline Clinical parameters & $\begin{array}{c}\text { Patients }(\mathrm{n}=15), \begin{array}{c}\text { Healthy controls } \\
\mathrm{n}(\mathrm{n}=15), \mathrm{n}(\%)\end{array} \\
\text { Sex range) }\end{array}$ & $6(40)$ \\
Male & $8(53.3)$ & $9(60)$ \\
Female & $7(46.7)$ & - \\
Age (years) & $64(54-72)$ & $60(52-70)$ \\
Cerebral ischemic risk & & - \\
factors & & - \\
Hypertension & $8(53.3)$ & - \\
Diabetes mellitus & $4(26.7)$ & 0 \\
Atrial Fibrillation & $5(33.3)$ & - \\
Hyperlipidemia & $6(40)$ & - \\
Coronary heart disease & $3(20)$ & 0 \\
Baseline median NIHSS & 8 & - \\
score & & \\
25th-75th percentile & $6-11$ & \\
Baseline DWI median & 18.58 & \\
volume (cm $\left.{ }^{3}\right)$ & $9.79-30.48$ & \\
25th-75th percentile $\left(\mathrm{cm}^{3}\right)$ & & \\
\hline
\end{tabular}

All continuous data are expressed as the median (range), and categorical variables are expressed as the n values (percentages). NIHSS, National Institute of Health Stroke Scale; DWI, diffusion weighted imaging.

dilution), VEGF (cat. no. 2463; Cell Signaling Technology, Inc.; 1:1,000 dilution) and total VEGFR2 (cat. no. 9698; Cell Signaling Technology, Inc.; 1:1,000 dilution) and $\beta$-actin (cat. no. sc-58673; Santa Cruz Biotechnology, Inc.; 1:2,000 dilution) were incubated with the membrane at $4^{\circ} \mathrm{C}$ overnight. Following incubation with anti-rabbit IgG $\left(\mathrm{H}+\mathrm{L}\right.$; DyLight ${ }^{\mathrm{TM}}$ 680 Conjugate; cat. no. 5366; Cell Signaling Technology, Inc.; 1:10,000 dilution), bands were detected using an enhanced chemiluminescence kit (GE Healthcare). The intensity of the bands of interest was analyzed by ImageJ software (version 1.46; National Institutes of Health).

Statistical analysis. Statistical analysis was performed using SPSS software (version 18.0; IBM Corp., Chicago, IL, USA). Data were presented as the mean \pm standard deviation. Student's t-test or one-way analysis of variance followed by Tukey post hoc test was used to analyze the difference among/between sample groups. $\mathrm{P}<0.05$ was considered to indicate a statistically significant difference.

\section{Results}

miR-451 is downregulated in the serum samples of patients with cerebral ischemia. To explore the potential involvement of miRNAs in CII, the present study performed miRNA microarray profiling in serum samples from patients with cerebral ischemia. The miRNA microarray identified 35 miRNAs that were upregulated and 22 miRNAs that were downregulated 
in the ischemia group when compared with the control group (Fig. 1A). Among them, miR-451 was the most significantly downregulated, and previous studies have revealed that miR-451 functions as a suppressor of angiogenesis in hepatocellular carcinoma (18) and human osteosarcoma (19), but little is known regarding its role in the regulation of angiogenesis in CII. Therefore, the present study decided to focus on miR-451 in CII for further study.

To confirm the microarray findings, the miR-451 expression levels were determined by RT-qPCR in all samples. miR-451 was significantly downregulated in the serum samples from patients with cerebral ischemic compared with the normal participants (Fig. 1B), which is consistent with the results observed following microarray analysis. All of these results suggest that the alterations in miR-451 expression may serve important roles in CII.

Overexpression of miR-451 suppresses the tube formation and migration of HUVECs under hypoxic conditions. To assess the biological role of miR-451 in angiogenesis, the present study applied HUVECs under hypoxic conditions to mimic ischemia in vitro $(20,21)$ and the expression of miR-451 was determined by RT-qPCR. As shown in Fig. 2A, hypoxia treatment induced a significant decrease in the expression of miR-451 in HUVECs, which reached a peak at $6 \mathrm{~h}$ and then raised to near basal levels by $12 \mathrm{~h}$. Therefore, the present study selected the $6 \mathrm{~h}$ time point as the subsequent experimental condition to study the influence of miR-451 on angiogenesis. As angiogenesis is defined as new microvessel formation via branching off from pre-existing vessels, which involves multi-step biological processes, including proliferation, migration and the formation of tube-like vascular structures (22-24), capillary-like tube formation and migration assays are normally used to evaluate angiogenesis in vitro (25). To further investigate the role of miR-451 in tube formation and migration, HUVECs were transfected with miR-451 mimics or mimics NC, followed by normoxic or hypoxia treatment. It was observed that the expression of miR-451 was enhanced following miR-451 mimics transfection under normoxia or hypoxia conditions (Fig. 2B). Furthermore, the tube formation assays demonstrated that overexpression of miR-451 significantly reduced the ability of HUVECs to form tubular structures during hypoxia (Fig. 2C). The Transwell assay revealed that the number of invaded cells was significantly reduced in miR-451 mimics group when compared with mimics NC group (Fig. 2D). In addition, the results also revealed that miR-451 mimics decreased the cell migration distance when compared with the mimics NC group (Fig. 2E).

Given the importance of the VEGF/VEGFR axis in angiogenesis following CII (26-29), the present study sought to determine whether miR-451 affects this axis in HUVECs. The results of western blotting revealed that the expressions of VEGF and p-VEGFR2 were markedly increased in HUVECs under hypoxic conditions, whereas this promotional effect was attenuated following treatment with miR-451 mimics (Fig. 2F), which indicated that the overexpression of miR-451 could inhibit the VEGF/VEGFR2 pathway.

Knockdown of miR-451 promotes the tube formation and migration of HUVECs under normoxic conditions. Next, to assess the effect of miR-145 inhibition on HUVECs migration and tubulogenesis under normoxic conditions, HUVECs were transfected with miR-451 inhibitor to decrease miR-451 expression and then under normoxic conditions for $6 \mathrm{~h}$. Following miR-451 inhibitor transfection, miR-451 expression was markedly decreased when compared with inhibitor NC (Fig. 3A). Then, the angiogenic properties of HUVECs were detected using tube formation, Transwell and wound healing assays. The tube formation assays revealed that knockdown of miR-451 promoted the ability of HUVECs to form tubular structures during normoxia (Fig. 3B). The Transwell assay revealed that the number of invading cells was significantly increased in the miR-451 inhibitor group when compared with the inhibitor NC group (Fig. 3C). In addition, miR-451 inhibitor also increased the cell migration distance when compared with the inhibitor NC group (Fig. 3D). All of these results indicated that low level miR-451 is beneficial to angiogenesis in HUVECs.

MIF is a direct target of $m i R-451$. To explore the molecular mechanisms by which miR-451 regulates the tube formation, invasion and migration of HUVECs, candidate target genes of miR-451 were computationally screened using TargetScan and PicTar algorithms. Among several predicted target genes, MIF, which has been reported to improve angiogenesis, was of interest due to its high scores in the two algorithms. As shown in Fig. 4A, miR-451 contained a sequence that was complementary to MIF. In addition, previous research has shown that MIF was a direct target of miR-451 in human osteosarcoma (30). However, the association between miR-451 and MIF in CII remains unclear. Firstly, the present study determined the expression of MIF in HUVECs under hypoxic conditions by RT-qPCR. As shown in Fig. 4B, hypoxia treatment caused a significant increase in the expression of MIF in HUVECs, which reached a peak at $6 \mathrm{~h}$ and declined to near basal levels by $12 \mathrm{~h}$. The expression levels of MIF in all serum samples were also determined and it was revealed that the expression of MIF was significantly increased in the ischemia group when compared with the normal group (Fig. 4C). To further confirm that MIF was negatively regulated by miR-451, the present study performed western blot analysis to determine the protein level of MIF. The expression of MIF at the protein level was significantly downregulated following the overexpression of miR-451 in HUVECs cells under hypoxic conditions, but upregulated following knockdown of miR-451 in HUVECs cells under normoxic conditions (Fig. 4D and E). To verify whether MIF is a direct target of miR-451, the 3'-UTR of MIF containing the WT or Mut miR-451 target sequences was cloned into the pmirGLO vector. Co-transfection was conducted with these reporter plasmids and miR-451 mimics, mimics NC, miR-451 inhibitor or inhibitor-NC into HUVECs, then luciferase activities were analyzed $48 \mathrm{~h}$ post-transfection. Luciferase reporter gene assays demonstrated that overexpression of miR-451 markedly repressed, while knockdown of miR-451 increased, the relative luciferase activity of constructs containing the WT MIF 3'-UTR. However, the luciferase activity of the reporter containing the mutant binding site was not altered (Fig. 4F). These data indicated that miR-451 regulates the expression of MIF in HUVECs. 
A

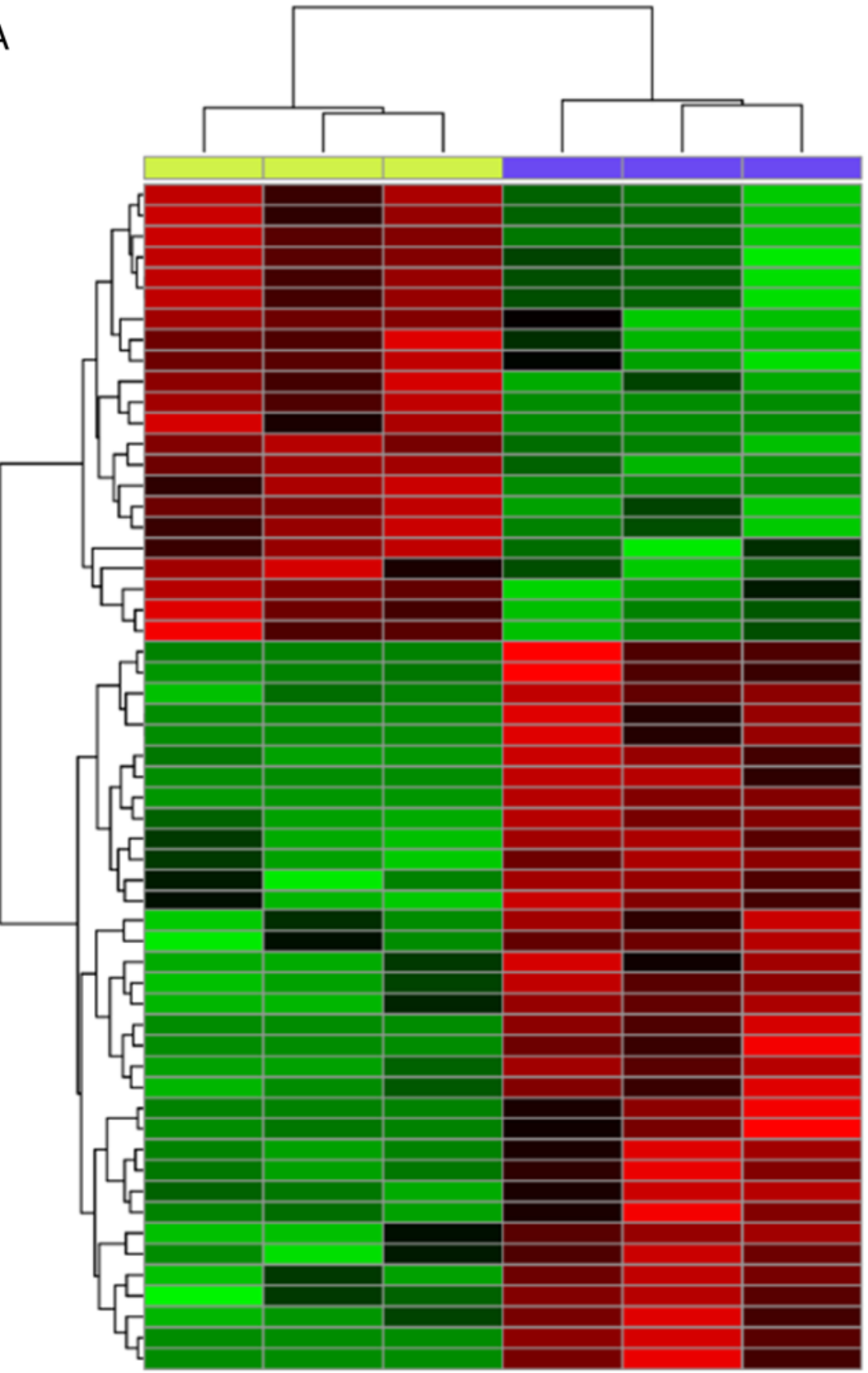
Group
$\square$ Normal
Ischemia

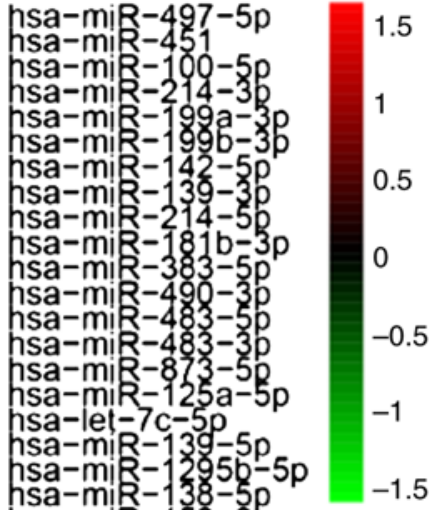

ha-miR-150-3p

hsa-mik-101-3p

hsa-miR-548 d-5

ha-mik-193a-3p

isa-miR-3127-3p

hsa-mik-58

isa-miR-18a-3p

hsa-miR- $6516-3 p$

hsa-miR-7641

hsa-mik-301a-3p

isa-miR-210-3p

ha-miR-425-5p.

hsa-miR-548ay-5p

ha-miR-58 -3p

isa-miR-3177-3p

isa-miR-93-3p

isa-mi $-5010-5 p$

hsa-m!

isa-miR-6 $316-3 p$

isa-mik-1246

hsa-mi R-2277-5p

isa-mik-548a-3p

ha-mi -151 a-3p

isa-miR-423-3p

hsa-mi 1276

hsa-miR-17-5p

hsa-mik-476 $2-5 p$

hsa-miR-128-1-5p

hsa-miR-5010-3p

sa-miR-3158-3p

hsa-miR-128-3p

isa-miR-3936

hsa-miR-643

B

$p<0.01$

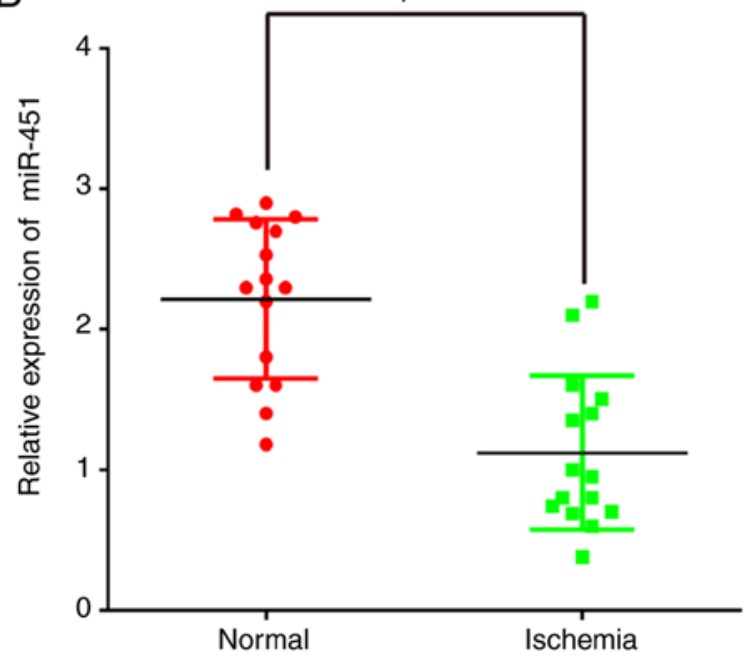

Figure 1. miR-451 is downregulated in serum samples from cerebral ischemic patients. (A) The hierarchical clustering of miRNAs differentially expressed in serum samples from cerebral ischemic patients compared with the normal group $(n=3)$. (B) miR-451 levels were measured in serum samples from cerebral ischemic patients $(\mathrm{n}=15)$ and normal participants $(\mathrm{n}=15)$ by reverse transcription-quantitative polymerase chain reaction. $\mathrm{P}<0.01$, as indicated. miR, microRNA. 


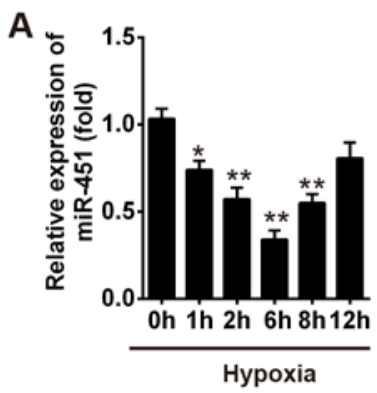

B

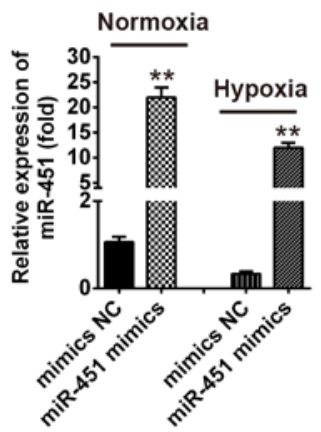

D
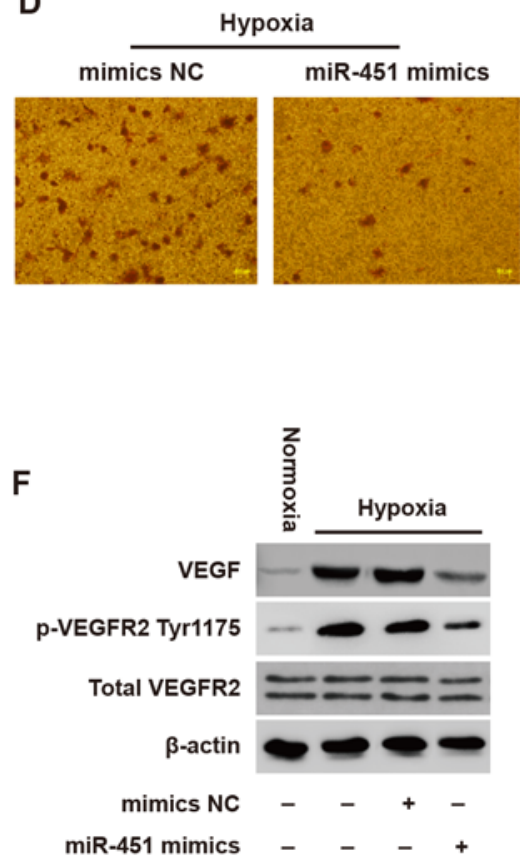

C
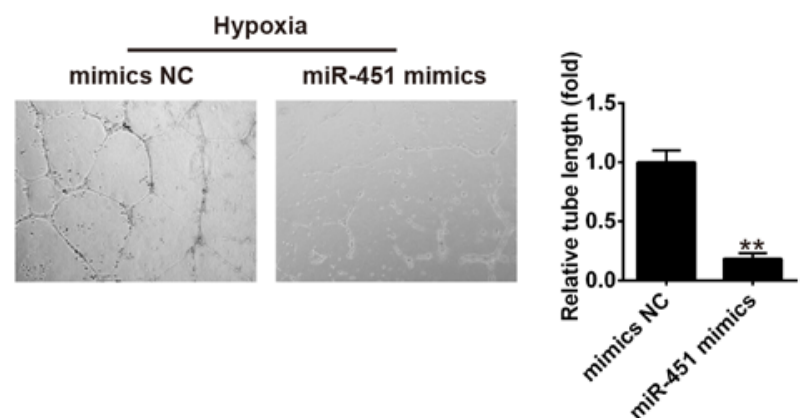

E
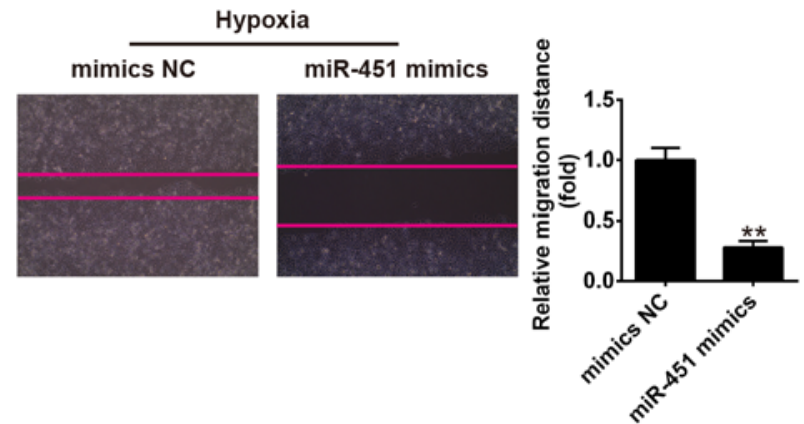

Figure 2. Overexpression of miR-451 suppresses the tube formation and migration of HUVECs under hypoxic conditions. (A) HUVECs were treated with hypoxia for the indicated time points. The relative expression level of miR- 451 was detected by RT-qPCR. ${ }^{*} \mathrm{P}<0.05$ and ${ }^{* * *} \mathrm{P}<0.01$ vs. control group $(0 \mathrm{~h})$. HUVECs were transfected with miR-451 mimics or mimics NC. Following $24 \mathrm{~h}$, cells were treated with normoxia or hypoxia for $6 \mathrm{~h}$. (B) The relative expression level of miR-451 was determined by RT-qPCR under normoxia or hypoxia conditions. All data are expressed as the mean \pm standard deviation, ${ }^{* *} \mathrm{P}<0.01 \mathrm{vs}$. mimics NC group. (C) The tube formation of HUVECs was measured by Matrigel assays (magnification, $\mathrm{x} 200$ ). All data are expressed as the mean \pm standard deviation. ${ }^{* *} \mathrm{P}<0.01$ vs. mimics NC group. (D) The invasive ability of HUVECs was measured by a Transwell assay (magnification, $\mathrm{x} 200$ ). All data are expressed as the mean \pm standard deviation. ${ }^{* *} \mathrm{P}<0.01$ vs. mimics NC group. (E) Cell migration distance was detected by the wound healing assay (magnification, $\mathrm{x} 200$ ). All data are expressed as the mean \pm standard deviation. ${ }^{* *} \mathrm{P}<0.01$ vs. mimics NC group. (F) The expressions of $\mathrm{p}-\mathrm{VEGFR} 2$ (Tyr1175), VEGF and total VEGFR 2 proteins were measured by western blotting. All data are expressed as the mean \pm standard deviation. ${ }^{*} \mathrm{P}<0.05$ and ${ }^{* *} \mathrm{P}<0.01$ vs. Normoxia group; ${ }^{\# \#} \mathrm{P}<0.01$, as indicated. RT-qPCR, reverse transcription-quantitative polymerase chain reaction; miR, microRNA; HUVECs, human umbilical vein endothelial cells; VEGFR, vascular endothelial growth factor receptor; VEGF, vascular endothelial growth factor; p-, phosphorylated; NC, negative control.

$M I F$ reverses the effects of $m i R-451$ on the regulation of angiogenesis in vitro. To investigate whether MIF was involved in the anti-angiogenesis effects of miR-451, the present study transfected pcDNA-MIF plasmids into HUVECs cells, followed by exposure to hypoxic conditions. Western blotting was conducted to examine whether MIF protein expression was effectively enhanced. As shown in Fig. 5A, pcDNA-MIF transfection significantly overexpressed MIF protein expression in HUVECs. In addition, miR-451 mimics inhibited tube formation capacity, invasive ability and migration distance, and its inhibitory effect was attenuated when MIF was overexpressed (Fig. 5B-D). To inhibit MIF protein expression, the present study transfected MIF siRNA into HUVECs, followed by exposure to normoxic conditions. Western blotting was also performed to examine whether MIF protein expression was effectively altered. As shown in Fig. 5E, MIF siRNA transfection significantly inhibited MIF protein expression in HUVECs. Furthermore, the miR-451 inhibitor promoted tube formation capacity, invasive ability and migration distance, and its promotional effect was reduced when MIF was knocked down (Fig. 5F-H). These data suggested that the miR-451/MIF axis may serve an important role in the regulation of angiogenesis in HUVECs.

\section{Discussion}

In the present study, miR-451 was downregulated in serum samples from patients with cerebral ischemic. In an in vitro model of CII, hypoxia treatment promoted the tube formation and migration of HUVECs, while this enhancement was attenuated when miR-451 was overexpressed. Similar to the hypoxia condition, knockdown of miR-451 promoted 
A

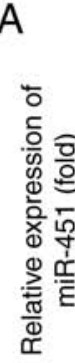

B

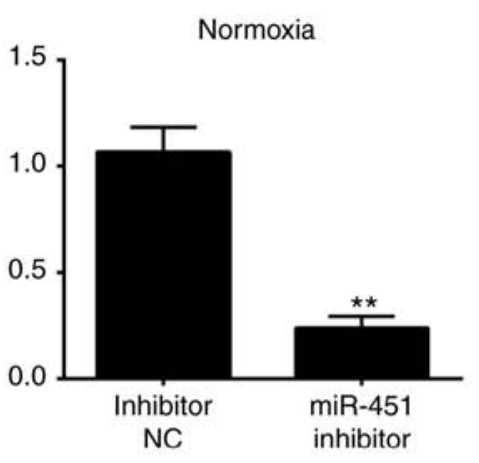

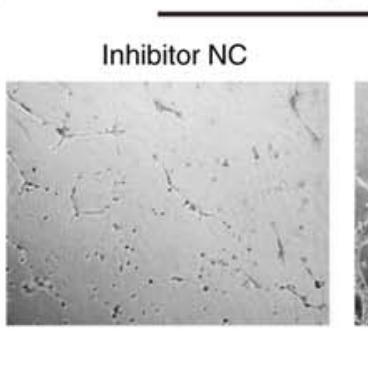

Normoxia

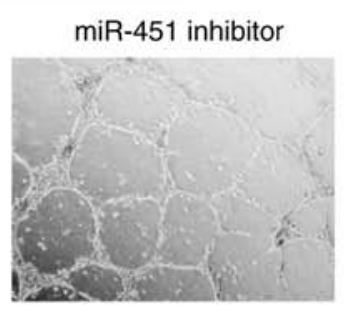

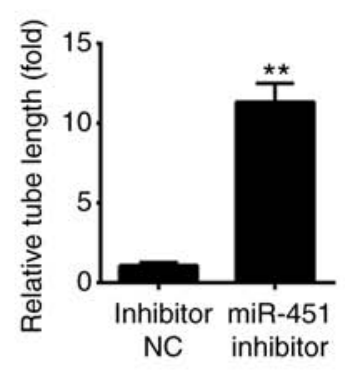

C

Normoxia

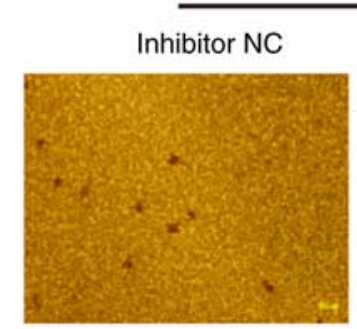

miR-451 inhibitor
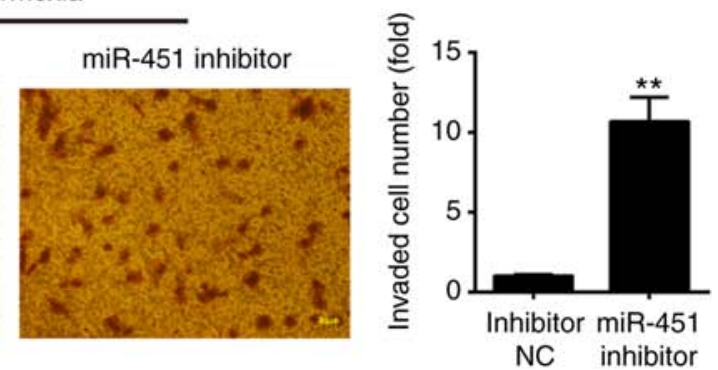

D
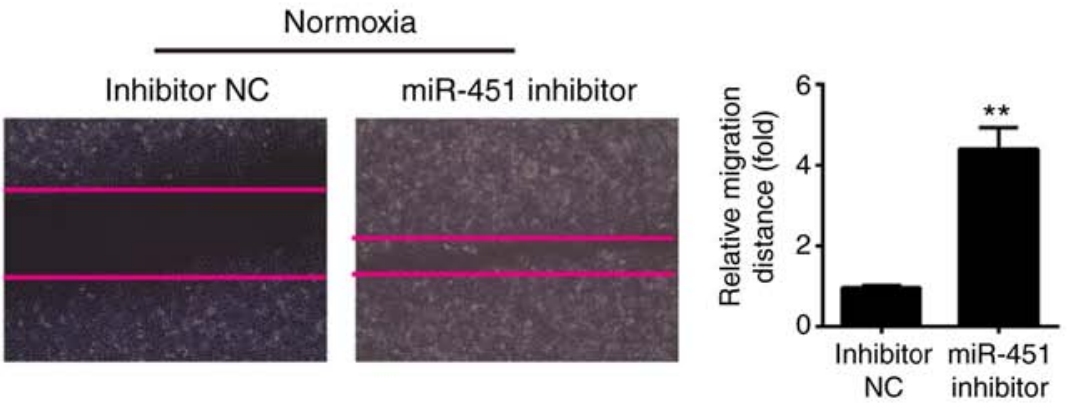

Figure 3. Knockdown of miR-451 promotes the tube formation and migration of HUVECs under normoxic conditions. HUVECs were transfected with miR-451 inhibitor or inhibitor NC. Following $24 \mathrm{~h}$, cells were treated with normoxia for $6 \mathrm{~h}$. (A) The relative expression level of miR-451 was determined by reverse transcription-quantitative polymerase chain reaction. (B) Tube formation of HUVECs was measured by Matrigel assays (magnification, $\mathrm{x} 200$ ). All data are expressed as the mean \pm standard deviation. ${ }^{* *} \mathrm{P}<0.01 \mathrm{vs}$. inhibitor NC group. (C) The invasive ability of HUVECs was measured by the Transwell assay (magnification, $\mathrm{x} 200$ ). All data are expressed as the mean \pm standard deviation. ${ }^{* *} \mathrm{P}<0.01$ vs. inhibitor NC group. (D) Cell migration distance was detected by the wound healing assay (magnification, $\mathrm{x} 200$ ). All data are expressed as the mean \pm standard deviation. ${ }^{* * *} \mathrm{P}<0.01$ vs. inhibitor NC group. HUVECs, human umbilical vein endothelial cells; miR, microRNA; NC, negative control.

the angiogenesis of HUVECs under normoxic condition. The present study further identified that MIF was a novel target of miR-451 and MIF mediated the effect of miR-451 on angiogenesis in HUVECs.

Recently, accumulating studies have revealed the important roles of miRNAs in regulating angiogenesis in cancer and other diseases (31-33). For example, Shi et al (34) revealed that inhibition of miR-103 could promote ischemic stroke angiogenesis and reduce infarction volume by enhancing VEGF in rats subjected to middle cerebral artery occlusion. Lou et al (20) demonstrated that upregulation of miR-210 can activate the Notch signaling pathway, which may contribute to angiogenesis following cerebral ischemia. Li et al (16) revealed that downregulation of miR-493 promoted angiogenesis in a rat model of ischemic stroke by targeting MIF. In another study, Yi et al (35) reported that miR-193-5p modulated angiogenesis through insulin-like growth factor 2 in type 2 diabetic cardiomyopathy. These results led to the conclusion that miRNAs may also serve an important role in the regulation of angiogenesis in CII. In the present study, a miRNA microarray screen was conducted, resulting in a set of differentially regulated miRNAs, including miR-451, which was downregulated in serum samples from cerebral ischemic patients. These results suggest that miR-451 may be involved in this pathological condition.

In recent years, emerging evidence has revealed that miR-451 serves important roles in the regulation of angiogenesis via effects on its target mRNA. For example, miR-451 inhibited cell migration and angiogenesis in human osteosarcoma by downregulating IL 6R (19). Liu et al (18) revealed that miR-451 inhibited angiogenesis in hepatocellular carcinoma by targeting the IL-6R-signal transducer and activator of transcription-3 signaling pathway. However, whether miR-451 has a similar mechanism in the regulation of angiogenesis following CII remains unknown. To further verify miR-451's role in angiogenesis following CII, the present study performed experiments with hypoxic HUVECs cells, which are always used to mimic CII. Subsequently, hypoxia treatment was 
A
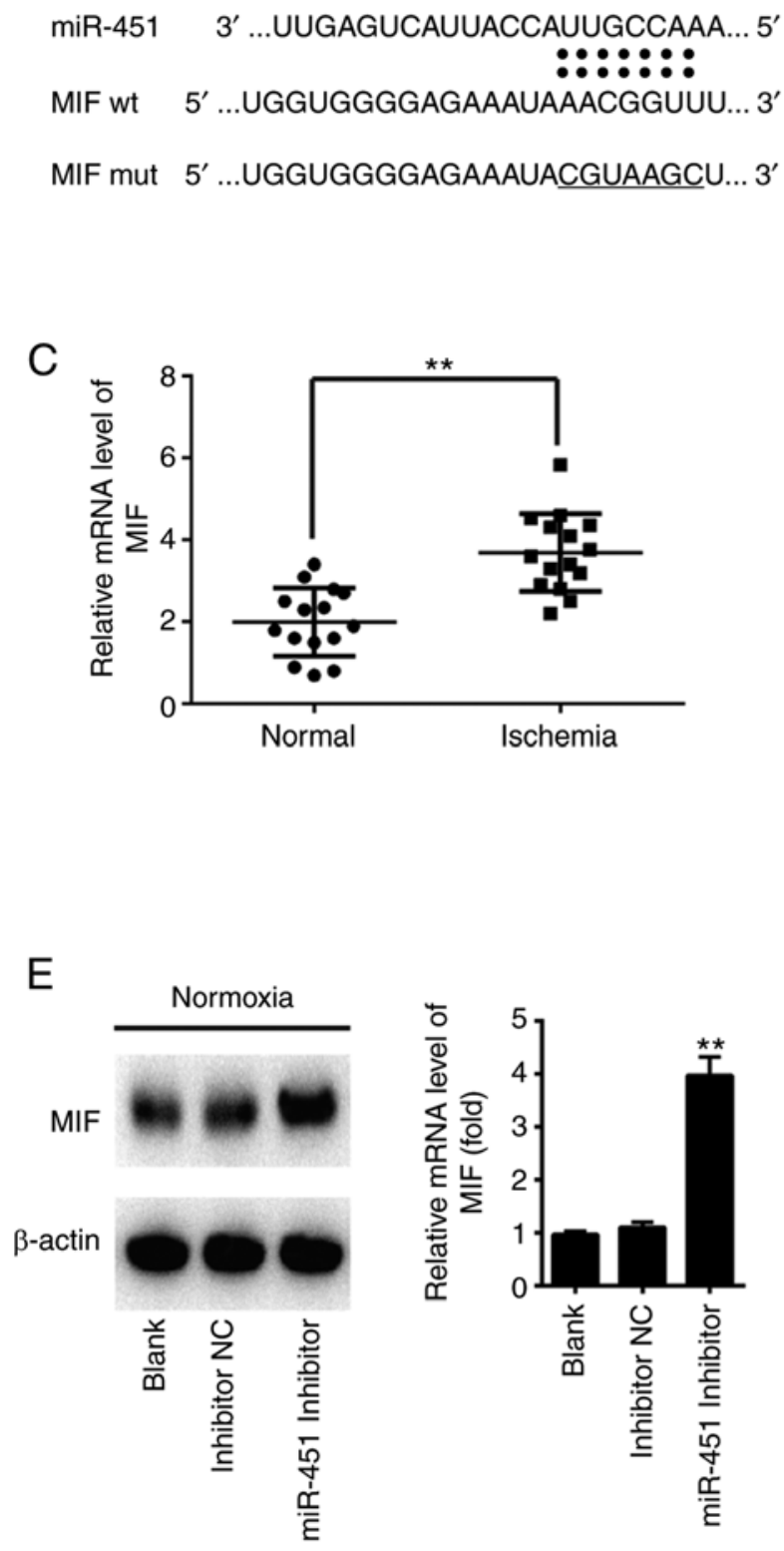

B
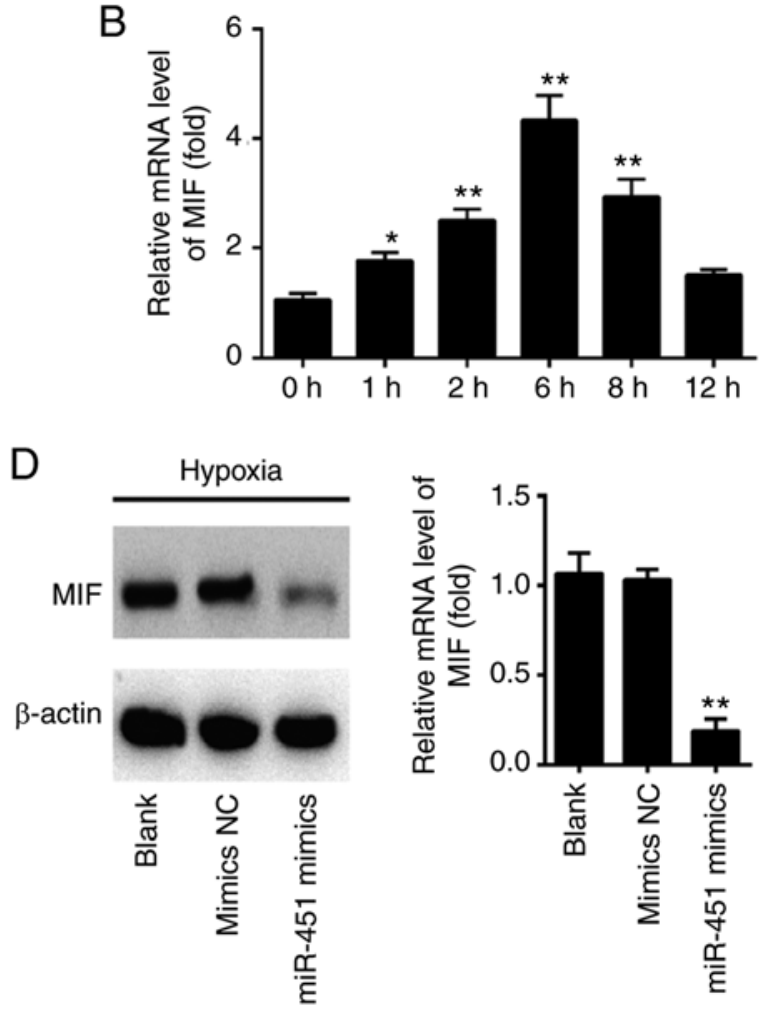

$\mathrm{F}$

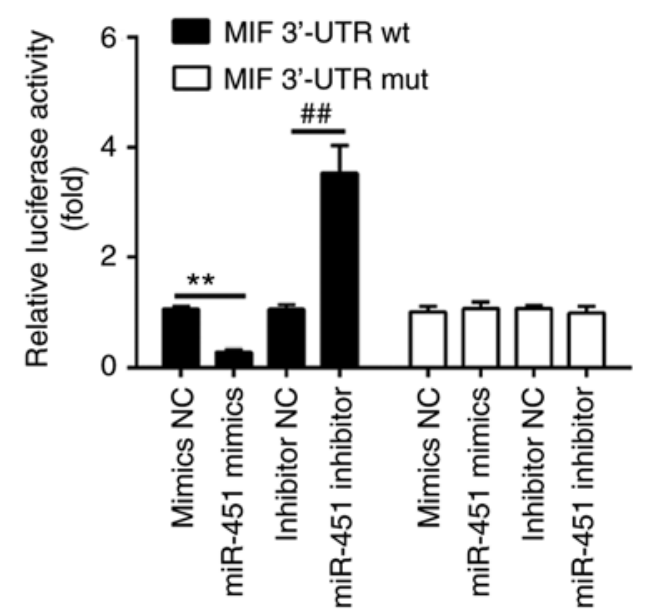

Figure 4. MIF is a direct target of miR-451. (A) Schematic of the MIF 3'UTR containing the miR-451 binding sites. (B) HUVECs were treated with hypoxia for the indicated time points. The relative expression level of MIF was detected by RT-qPCR. ${ }^{*} \mathrm{P}<0.05$ and ${ }^{* *} \mathrm{P}<0.01$ vs. control group $(0 \mathrm{~h})$. (C) miR- 451 level was measured in serum samples from cerebral ischemic patients $(n=15)$ and normal participants $(n=15)$ by RT-qPCR. ${ }^{* *} \mathrm{P}<0.01$, as indicated. (D) HUVECs were transfected with miR-451 mimics or mimics NC. Following $24 \mathrm{~h}$, cells were treated with hypoxia for $6 \mathrm{~h}$. The expression levels of MIF protein were determined by western blotting. ${ }^{* *} \mathrm{P}<0.01$ vs. mimics NC group. (E) HUVECs were transfected with miR-451 inhibitor or inhibitor NC. Following $24 \mathrm{~h}$, cells were treated with normoxia for $6 \mathrm{~h}$. The expression levels of MIF protein was determined by western blotting. ${ }^{* *} \mathrm{P}<0.01$ vs. inhibitor NC group. (F) Relative luciferase activity in HUVECs co-transfected with wild-type or mutant-type 3'UTR MIF reporter plasmids and miR-451 or miR-NC. All data are expressed as the mean \pm standard deviation. ${ }^{* *} \mathrm{P}<0.01$, as indicated; ${ }^{\# /} \mathrm{P}<0.01$, as indicated. RT-qPCR, reverse transcription-quantitative polymerase chain reaction; MIF, migration inhibitory factor; HUVECs, human umbilical vein endothelial cells; miR, microRNA; NC, negative control; UTR, untranslated region; wt, wild-type; mut, mutant.

revealed to reduce the expression of miR-451 in HUVECs and enhanced miR-451 expression attenuated the tube formation and migration of HUVECs under hypoxic conditions. Notably, knockdown of miR-451 promoted the angiogenesis of HUVECs under normoxic conditions, which is consistent with the results of hypoxia treatment. Previous studies have reported the involvement of the VEGF/VEGFR axis in angiogenesis following CII. For example, For example, Marti et al (29) indicated that VEGF and the VEGF receptors (VEGFR-1 and
VEGFR-2) are upregulated by hypoxia in the brain following cerebral ischemia, which mediated the angiogenic response in the ischemic border zone and extended towards the core region of the infarcted area. In the present study, overexpression of miR-451 could inhibit the expressions of VEGF and VEGFR2 via hypoxia, which indicated that miR-451 may regulate angiogenesis through the VEGF/VEGFR2 pathway.

Many studies have indicated that several angiogenic factors are significantly induced following CII, such as VEGF 

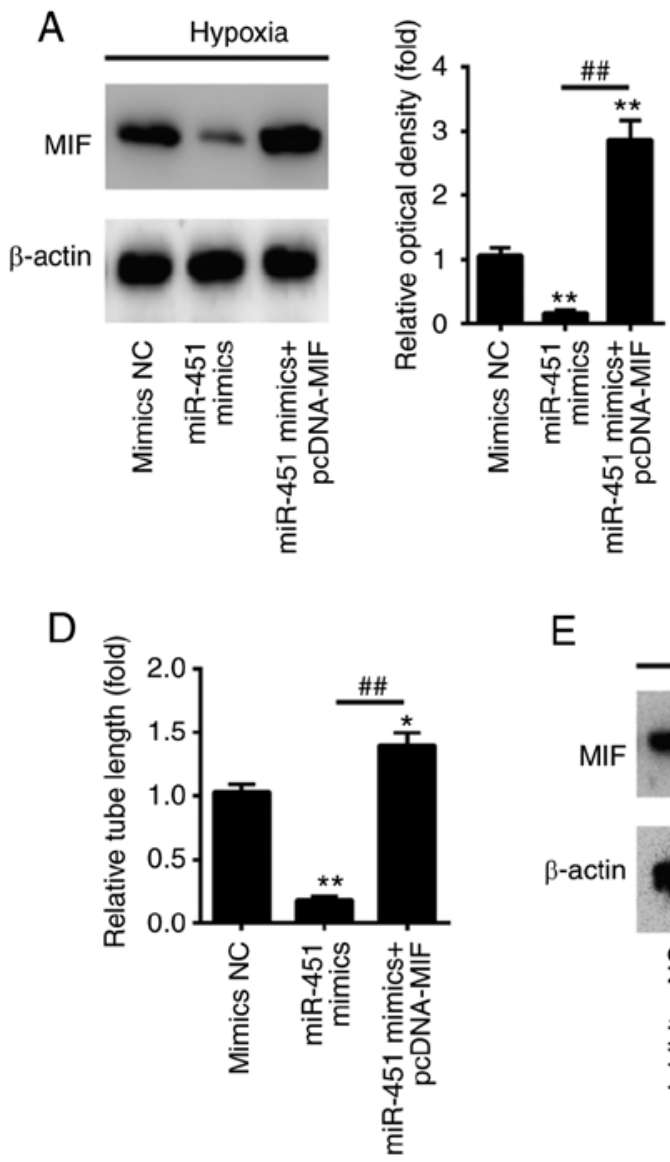

B

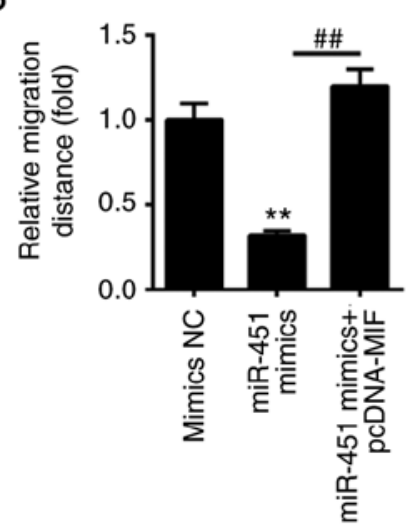

E

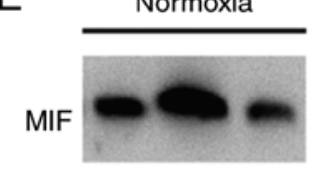

$\beta$-actin
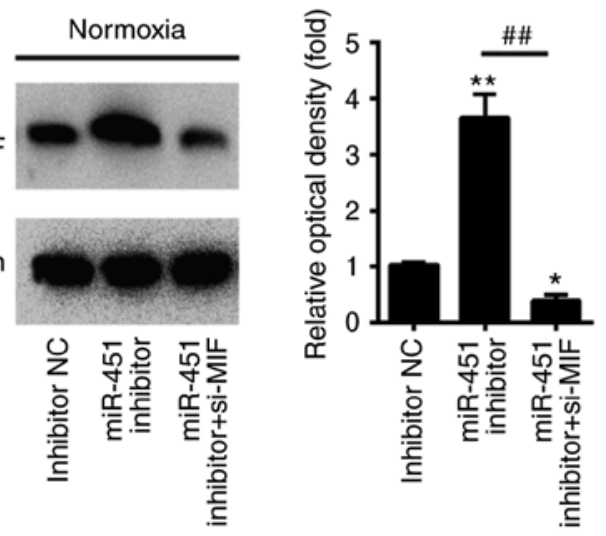

C

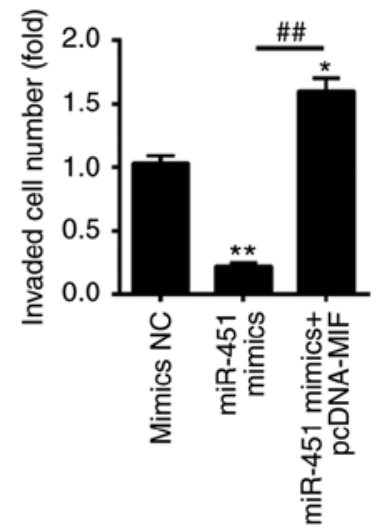

$\mathrm{F}$

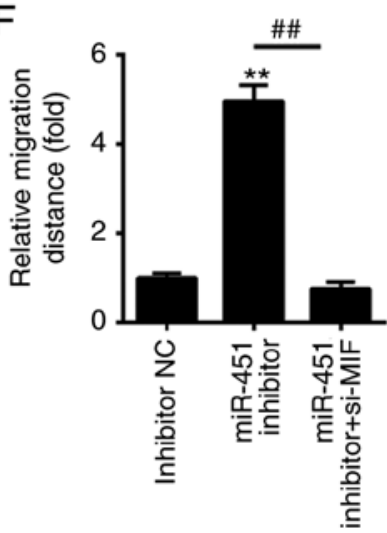

\section{G}

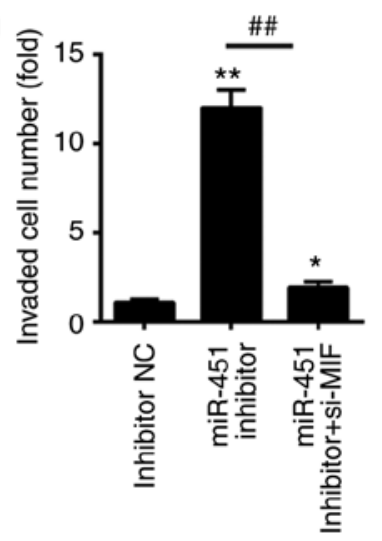

$\mathrm{H}$

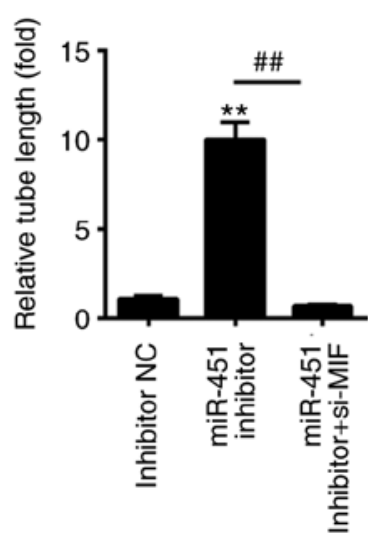

Figure 5. miR-451 inhibits angiogenesis by targeting MIF. HUVECs were co-transfected with miR-451 mimics or pcDNA-MIF. Following 24 h, cells were treated with hypoxia for $6 \mathrm{~h}$. (A) The expression levels of MIF protein were determined by western blotting. (B) The cell migration distance was detected by the wound healing assay, (C) the penetrating ability of HUVECs was measured by the Transwell assay, and (D) the tube formation of HUVECs was measured by Matrigel assays. ${ }^{*} \mathrm{P}<0.05$ and ${ }^{* *} \mathrm{P}<0.01$ vs. mimics NC group; ${ }^{\# /} \mathrm{P}<0.01$ vs miR-451 mimics group. (E-H) HUVECs were co-transfected with the miR-451 inhibitor or si-MIF. Following 24 h, cells were treated with hypoxia for $6 \mathrm{~h}$. (E) The expression levels of MIF protein were determined by western blotting. (F) The cell migration distance was detected by the wound healing assay, (G) the penetrating ability of HUVECs was measured by the Transwell assay and $(\mathrm{H})$ the tube formation of HUVECs was measured by Matrigel assays. ${ }^{*} \mathrm{P}<0.05$ and ${ }^{* *} \mathrm{P}<0.01$ vs. inhibitor $\mathrm{NC}$ group; ${ }^{\# \#} \mathrm{P}<0.01$, as indicated. All data are expressed as the mean \pm standard deviation. miR, microRNA; NC, negative control; MIF, migration inhibitory factor; HUVECs, human umbilical vein endothelial cells; si-, small interfering RNA.

and angiopoietin-2 (36,37). To determine the mechanism by which miR-451 functions in angiogenesis following CII, the present study predicted its target genes through bioinformatics analysis. MIF, a well-known angiogenic regulator, has been implicated in angiogenesis $(9,38)$. For example, Liao et al (8) demonstrated that MIF contributed to lymph node metastasis by inducing angiogenesis via the upregulation of IL-8 expression in head and neck squamous cell carcinoma. Notably, a recent study indicated that MIF enhanced microvessel-like tube formation by promoting the expression of angiogenesis associated genes in endothelial cells (39). MIF was also involved in CII (11). Loss of MIF exacerbated injury in the female brain following experimental stroke, which was independent of changes in pro-inflammatory cytokine levels (40). In the present study, MIF expression was increased in serum samples and HUVECs exposed to hypoxia, which led to the 
hypothesis that miR-451 targets MIF to impair angiogenesis following CII. To test this hypothesis, the present study performed rescue experiments by ectopically expressing a miR-resistant variant of MIF. It was observed that enforced expression of MIF restored the tube formation and migration of HUVECs reduced by miR-451 overexpression under hypoxic conditions, while silencing MIF reversed the promotional effects, triggered by miR-451 knockdown, on the angiogenesis of HUVECs under normoxic conditions. Collectively, miR-451 acts as a negative regulator of angiogenesis in the HUVEC model largely through downregulation of MIF.

Rapid and accurate diagnosis of CII is critical to enable the development of appropriate treatment. Although the medical advancements are made frequently, effective diagnosis of acute CII is still lacking (41). Therefore, there is a requirement for a more rapid and simple tool for CII diagnosis. Circulating miRNAs can serve as indicators for the diagnosis, progression and prognosis of various diseases (42-44). Thus, miRNA levels in peripheral blood may be closely associated when under conditions of CII. In the present study, differential expression of several miRNAs in the serum samples of cerebral ischemic patients were observed when compared with healthy subjects; in particular, the expression of miR-451 was downregulated. Through further study, it was verified that the level of miR-541 was decreased in HUVECs under hypoxic conditions when compared with those under normoxic conditions. Notably, the present results revealed that $\mathrm{miR}-451$ served an important role in angiogenesis following CII. However, miR-451 was identified as the most significantly downregulated miRNA in serum samples, but it was not detected in endothelial tissue samples. Therefore, it is difficult to distinguish whether the downregulation of miR-451 in serum was derived from it in endothelial cells in the same lesion or not. In the future, the group will perform the relevant research in endothelial tissue samples.

In conclusion, the present study has provided evidence that miR-451 expression was downregulated following cerebral ischemia. Downregulation of miR-451 could be beneficial for angiogenesis by increasing MIF expression in hypoxic HUVECs. These results may highlight the importance of miR-451 in treating CII. Further study of these mechanisms has the potential to lead to targeted clinical therapy.

\section{Acknowledgements}

Not applicable.

\section{Funding}

No funding was received.

\section{Availability of data and materials}

All data generated or analyzed during this study are included in this published article.

\section{Authors' contributions}

QL, YL, DZ, HG and XG performed the experiments, contributed to data analysis and wrote the paper. YL, DZ, HG and XG analyzed the data. QL conceived and designed the study, and contributed to data analysis and the experimental materials. All authors read and approved the final manuscript.

\section{Ethics approval and consent to participate}

All individuals provided written informed consent for the use of human specimens for clinical research. The present study was approved by Workers' Hospital of Tangshan City (Hebei, China).

\section{Patient consent for publication}

Not applicable.

\section{Competing interests}

The authors declare that they have no competing interests.

\section{References}

1. Johnston SC, Mendis S and Mathers CD: Global variation in stroke burden and mortality: Estimates from monitoring, surveillance, and modelling. Lancet Neurol 8: 345-354, 2009.

2. Xu AD, Wang YJ, Wang DZ and Chinese Stroke Therapy Expert Panel for Intravenous Recombinant Tissue Plasminogen Activator: Consensus statement on the use of intravenous recombinant tissue plasminogen activator to treat acute ischemic stroke by the chinese stroke therapy expert panel. CNS Neurosci Ther 19: 543-548, 2013.

3. Lo EH, Dalkara T and Moskowitz MA: Mechanisms, challenges and opportunities in stroke. Nat Rev Neurosci 4: 399-415, 2003.

4. Semenza GL: Vasculogenesis, angiogenesis, and arteriogenesis: Mechanisms of blood vessel formation and remodeling. J Cell Biochem 102: 840-847, 2007.

5. Choudhary S, Hegde P, Pruitt JR, Sielecki TM, Choudhary D, Scarpato K, Degraff DJ, Pilbeam CC and Taylor JA 3rd: Macrophage migratory inhibitory factor promotes bladder cancer progression via increasing proliferation and angiogenesis. Carcinogenesis 34: 2891-2899, 2013.

6. Asare Y, Schmitt M and Bernhagen J: The vascular biology of macrophage migration inhibitory factor (MIF). Expression and effects in inflammation, atherogenesis and angiogenesis. Thromb Haemost 109: 391-398, 2013.

7. Rassaf T, Weber $\mathrm{C}$ and Bernhagen J: Macrophage migration inhibitory factor in myocardial ischaemia/reperfusion injury. Cardiovasc Res 102: 321-328, 2014.

8. Liao B, Zhong BL, Li Z, Tian XY, Li Y and Li B: Macrophage migration inhibitory factor contributes angiogenesis by up-regulating IL-8 and correlates with poor prognosis of patients with primary nasopharyngeal carcinoma. J Surg Oncol 102: 844-851, 2010.

9. Girard E, Strathdee C, Trueblood E and Queva C: Macrophage migration inhibitory factor produced by the tumour stroma but not by tumour cells regulates angiogenesis in the B16-F10 melanoma model. Br J Cancer 107: 1498-1505, 2012.

10. Wang L, Zis O, Ma G, Shan Z, Zhang X, Wang S, Dai C, Zhao J, Lin Q, Lin S and Song W: Upregulation of macrophage migration inhibitory factor gene expression in stroke. Stroke 40: 973-976, 2009.

11. Zis O, Zhang S, Dorovini-Zis K, Wang L and Song W: Hypoxia signaling regulates macrophage migration inhibitory factor (MIF) expression in stroke. Mol Neurobiol 51: 155-167, 2015.

12. Ambros V: The functions of animal microRNAs. Nature 431: 350-355, 2004.

13. Bartel DP: MicroRNAs: Genomics, biogenesis, mechanism, and function. Cell 116: 281-297, 2004.

14. Liu Z, Yang D, Xie P, Ren G, Sun G, Zeng X and Sun X: MiR-106b and MiR-15b modulate apoptosis and angiogenesis in myocardial infarction. Cell Physiol Biochem 29: 851-862, 2012.

15. Zhao WJ, Zhang HF and Su JY: Downregulation of microRNA-195 promotes angiogenesis induced by cerebral infarction via targeting VEGFA. Mol Med Rep 16: 5434-5440, 2017. 
16. Li Q, He Q, Baral S, Mao L, Li Y, Jin H, Chen S, An T, Xia Y and $\mathrm{Hu}$ B: MicroRNA-493 regulates angiogenesis in a rat model of ischemic stroke by targeting MIF. FEBS J 283: 1720-1733, 2016.

17. Livak KJ and Schmittgen TD: Analysis of relative gene expression data using real-time quantitative PCR and the 2(-Delta Delta C(T)) method. Methods 25: 402-408, 2001.

18. Liu X, Zhang A, Xiang J, Lv Y and Zhang X: miR-451 acts as a suppressor of angiogenesis in hepatocellular carcinoma by targeting the IL-6R-STAT3 pathway. Oncol Rep 36: 1385-1392, 2016.

19. Liu SY, Deng SY, He YB and Ni GX: miR-451 inhibits cell growth, migration and angiogenesis in human osteosarcoma via down-regulating IL 6R. Biochem Biophys Res Commun 482: 987-993, 2017

20. Lou YL, Guo F, Liu F, Gao FL, Zhang PQ, Niu X, Guo SC, Yin JH, Wang Y and Deng ZF: miR-210 activates notch signaling pathway in angiogenesis induced by cerebral ischemia. Mol Cell Biochem 370: 45-51, 2012.

21. Li L, Wang M, Mei Z, Cao W, Yang Y, Wang Y and Wen A: IncRNAs HIF1A-AS2 facilitates the up-regulation of HIF-1a by sponging to miR-153-3p, whereby promoting angiogenesis in HUVECs in hypoxia. Biomed Pharmacother 96: 165-172, 2017.

22. Ding G, Jiang Q, Li L, Zhang L, Zhang ZG, Ledbetter KA Gollapalli L, Panda S, Li Q, Ewing JR and Chopp M: Angiogenesis detected after embolic stroke in rat brain using magnetic resonance T2*WI. Stroke 39: 1563-1568, 2008.

23. Ruan L, Wang B, ZhuGe Q and Jin K: Coupling of neurogenesis and angiogenesis after ischemic stroke. Brain Res 1623: 166-173, 2015.

24. Venna VR, Li J,Hammond MD, Mancini NS and McCullough LD: Chronic metformin treatment improves post-stroke angiogenesis and recovery after experimental stroke. Eur J Neurosci 39 2129-2138, 2014

25. He QW, Xia YP, Chen SC, Wang Y, Huang M, Huang Y, Li JY, Li YN, Gao Y, Mao L, et al: Astrocyte-derived sonic hedgehog contributes to angiogenesis in brain microvascular endothelial cells via RhoA/ROCK pathway after oxygen-glucose deprivation. Mol Neurobiol 47: 976-987, 2013.

26. Sun Y, Jin K, Xie L, Childs J, Mao XO, Logvinova A and Greenberg DA: VEGF-induced neuroprotection, neurogenesis, and angiogenesis after focal cerebral ischemia. J Clin Invest 111: $1843-1851,2003$

27. Kaya D, Gursoy-Ozdemir Y, Yemisci M, Tuncer N, Aktan S and Dalkara T: VEGF protects brain against focal ischemia without increasing blood--brain permeability when administered intracerebroventricularly. J Cereb Blood Flow Metab 25: 1111-1118, 2005

28. Feng Y, Rhodes PG and Bhatt AJ: Neuroprotective effects of vascular endothelial growth factor following hypoxic ischemic brain injury in neonatal rats. Pediatr Res 64: 370-374, 2008.

29. Marti HJ, Bernaudin M, Bellail A, Schoch H, Euler M, Petit E and Risau W: Hypoxia-induced vascular endothelial growth factor expression precedes neovascularization after cerebral ischemia. Am J Pathol 156: 965-976, 2000.

30. Liu W, Liu SY, He YB, Huang RL, Deng SY, Ni GX and Yu B: MiR-451 suppresses proliferation, migration and promotes apoptosis of the human osteosarcoma by targeting macrophage migration inhibitory factor. Biomed Pharmacother 87: 621-627, 2017.
31. Wang S and Olson EN: AngiomiRs--key regulators of angiogenesis. Curr Opin Genet Dev 19: 205-211, 2009.

32. Suarez Y and Sessa WC: MicroRNAs as novel regulators of angiogenesis. Circ Res 104: 442-454, 2009.

33. Kuehbacher A, Urbich C and Dimmeler S: Targeting microRNA expression to regulate angiogenesis. Trends Pharmacol Sci 29: 12-15, 2008.

34. Shi FP, Wang XH, Zhang HX, Shang MM, Liu XX, Sun HM and Song YP: MiR-103 regulates the angiogenesis of ischemic stroke rats by targeting vascular endothelial growth factor (VEGF). Iran J Basic Med Sci 21: 318-324, 2018.

35. Yi F, Shang Y, Li B, Dai S, Wu W, Cheng L and Wang X MicroRNA-193-5p modulates angiogenesis through IGF2 in type 2 diabetic cardiomyopathy. Biochem Biophys Res Commun 491: 876-882, 2017.

36. Chen J, Zhang C, Jiang H, Li Y, Zhang L, Robin A, Katakowski M, Lu M and Chopp M: Atorvastatin induction of VEGF and BDNF promotes brain plasticity after stroke in mice. J Cereb Blood Flow Metab 25: 281-290, 2005

37. Gui C, Li SK, Nong QL, Du F, Zhu LG and Zeng ZY: Changes of serum angiogenic factors concentrations in patients with diabetes and unstable angina pectoris. Cardiovasc Diabetol 12: 34, 2013.

38. Kanzler I, Tuchscheerer N, Steffens G, Simsekyilmaz S, Konschalla S, Kroh A, Simons D, Asare Y, Schober A, Bucala R, et al: Differential roles of angiogenic chemokines in endothelial progenitor cell-induced angiogenesis. Basic Res Cardiol 108: 310, 2013.

39. Shan ZX, Lin QX, Yang M, Zhang B, Zhu JN, Mai LP, Deng CY, Liu JL, Zhang YY, Lin SG and Yu XY: Transcription factor Ap-1 mediates proangiogenic MIF expression in human endothelial cells exposed to Angiotensin II. Cytokine 53: 35-41, 2011.

40. Turtzo LC, Li J, Persky R, Benashski S, Weston G, Bucala R, Venna VR and McCullough LD: Deletion of macrophage migration inhibitory factor worsens stroke outcome in female mice. Neurobiol Dis 54: 421-431, 2013

41. Zhou X, Su S, Li S, Pang X, Chen C, Li J and Liu J: MicroRNA-146a down-regulation correlates with neuroprotection and targets pro-apoptotic genes in cerebral ischemic injury in vitro. Brain Res 1648: 136-143, 2016.

42. Shalaby T and Grotzer MA: Tumor-associated CSF MicroRNAs for the prediction and evaluation of CNS malignancies. Int J Mol Sci 16: 29103-29119, 2015.

43. Yu X and Li Z: Serum microRNAs as potential noninvasive biomarkers for glioma. Tumour Biol 37: 1407-1410, 2016.

44. Yue X, Lan F, Hu M, Pan Q, Wang Q and Wang J: Downregulation of serum microRNA-205 as a potential diagnostic and prognostic biomarker for human glioma. J Neurosurg 124: 122-128, 2016.

This work is licensed under a Creative Commons Attribution-NonCommercial-NoDerivatives 4.0 International (CC BY-NC-ND 4.0) License. 